\title{
CONCEPTUAL DESIGN OF AN AUTONOMOUS RESCUE BOT FOR ASSISTANCE DURING NATURAL DISASTER RESCUE OPERATIONS
}

\section{NAMAN SHARMA, HARSH ALGHARE \& APOORVA KAUSHIK SINGH}

Department of Mechanical Engineering, Vellore Institute of Technology, Vellore, Tamil Nadu, India

The primary problem for which the bot is designed is to make an autonomous gadget capable of handling issues of natural disaster by finding the trapped victims and making a clear and safe path for the rescue workers to reach ground zero. It can be implemented during earthquakes, building collapse and other land based disasters. Various components have been considered during Bot designing to make it autonomous and perform functions without human intervention. A rocker-bogie mechanism allows easy movement on rough terrain. Robotic arms with multiple DOF allows easy approach to difficult places for lifting and moving objects. A remote control system in case the autonomous system fails, 3 wide angle cameras for side viewing and a High definition camera for head vision as well as 360 degree view. Loader for removing debris near the lower chassis. New concept puncture proof tires implemented using additive manufacturing. Tubular space frame manufactured using square hollow tubes with implementation of DFMA principles. Fully equipped with sensors like LiDAR, RADAR, SONAR, Stereo Camera, IMU, GPS using a distinct software architecture which handles the task of perception, mapping, control and surveillance. All these together minimise the risk faced by rescue workers during rescue operations.

KEYWORDS: Autonomous; Rescue, Natural Disaster; Defence, Robots; Surveillance \& Autodesk Fusion 360
\end{abstract}

Received: Jun 09, 2020; Accepted: Jun 29, 2020; Published: Jul 23, 2020; Paper Id.: IJMPERDJUN2020460

\section{INTRODUCTION}

\subsection{Problem Identification and Complications Faced During Rescue Operations}

Natural disaster is an unprecedented occurrence that can cause severe damage to life and infrastructure that occurs in different forms such as floods, earthquakes, tornadoes, etc. Focusing at the case of Earthquakes various damages happen like Building, bridge collapse, etc. which lead to people in that scenario getting entrapped in the multiple layers of debris. This is where the work of Rescuers comes into place where they have to save the trapped victims and get them out safely but at the cost of danger to their own lives. There are various complications that occur during a Rescue operation after an earthquake like the terrain becomes highly uneven which makes it difficult for the rescue workers to reach all affected areas. Big chunks of concrete and piled up one on another makes Rescuers incapable of moving them to pave way. Entering a collapse site can lead to further damages and possible gas leaks can lead to suffocation and fire hazards which is highly unfavourable and thus needs a beforehand scan of the whole area.

\section{LITERATURE REVIEW}

"Networked aerial-ground robot system with distributed task allocation for disaster management" - This paper presents applications and network of surveillance and emergency situation management. It also presents communicationbetween aerial and ground robots and how they coordinate together for execution of assigned 
missions. The robot team provides data related to transportation and communicates via wireless sensor networks during the mission. The aerial and ground robots are responsible for fire hazard detection and extinguishing [1]. "SENEKA - Sensor Network with Mobile Robots for Disaster Management" - This paper considers situations of present developed societies which are fully capable of tackling disasters but being completely unpredictable in nature disasters like Earthquakes can lead to great loss of life and infrastructure. In dangerous locations like Nuclear power plants which are already in continual danger of radiation leakages or explosions and therefore natural factors like earthquakes can lead to catastrophe. Rescuers have to respond to these locations rapidly to secure trapped workers.[2]. "Stereo Vision based indoor/outdoor Navigation for Flying Robots" - Using portable robots in real disaster management situations requires the structure to provide a firm degree of autonomy. In many situations a steady, high bandwidth radio link amid the robot and a ground station cannot be sure. Besides, external triangulation aids such as GPS are untrustworthy in areas such as urban ravines, or not available inside buildings. Therefore, at least sensor statistics needed for benign navigation has to be processed on-board the system. Being an autonomous system minimizes human error and the functioning solely depends on the functionality of the onboard processing software architecture [3]. "Workflow Study on Human-Robot Interaction in USAR" - This paper includes data from field trials performed using two tactical mobile robots in collaboration with expert rescue workers. Mobile robots have been added as a valuable asset in urban rescue operations, mitigating any further natural disaster. A practical approach has been used here by actually testing the robots and their working capabilities in the field in order to mark them fit for real-time rescue operations where a human-robot interaction is possible. [4]

\section{METHODOLOGY}

Keeping in mind to have least human intervention during a rescue operation, it was necessary to implement features in the bot which will perform functions expected to be performed by a human rescue worker and hence, sensors are incorporated.

Sensors used and their function:

- Perception - Light Detection and Ranging (LiDAR), Radio Detection and Ranging (RADAR), Sound Navigation and Ranging(SONAR)

- $\quad$ Mapping - Global Positioning System(GPS), Inertial Measurement Unit(IMU)

- Object Detection and Response - Thermographic Camera(CMOS), Stereo Camera

A multi-wheel mechanism for easy movement of the robot on rough terrain has been incorporated in order to eliminate the use of suspension system as well as to increase the surface area in contact with the ground. Robotic arm with multiple degrees of freedom has been designed to allow easy approach in difficult places as well as for the function of debris removal. Optical devices have been used in order to provide "eyes" to the robot in order to make the robot capable of carrying out operations autonomously. Conceptual design and 3D modeling is done using Autodesk Fusion 360 software where additional Add-ins which is Bommer was used to come up with a conceptual design with the part manufacturing and cost estimates and additive manufacturing process simulation is done using UltimakerCura 4.1.

\section{PARTS AND MODEL}

\subsection{Tubular Space Frame Chassis}

The Tubular spaceframe chassis for the bot has been modeled and manufactured using Square hollow tubes as shown in Figure 1 which are available easily, therefore manufacturing is easy and convenient. The advantages of these tubes are that 
they are cost-effective, assembly and attachment of various components can be done easily and these provide weight reduction without much compromise in stiffness compared to a sheet metal structure. A proper manufacturing Jig was constructed for proper positioning of each tube and to minimise the error between model and manufactured part shown in Figure 2.
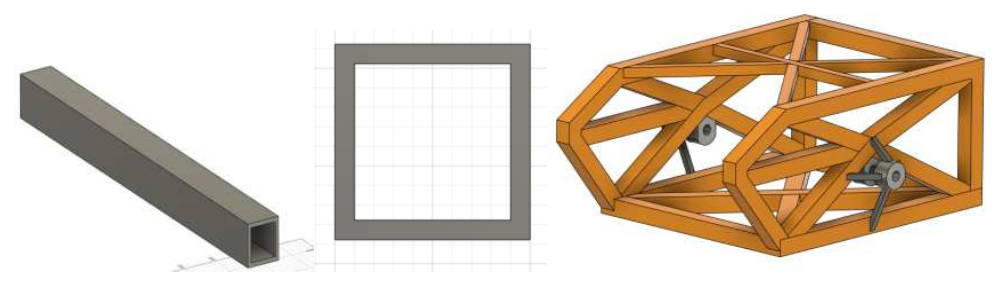

Figure 1: Square tube used in chassis and cross-section Figure 2: Chassis member arrangement

\subsection{Rocker-Bogie Suspension Mechanism}

Rocker-Bogie mechanism is designed for the bot to climb obstacles such as rocks, debris, etc. considering a case upto twice the diameter of the tires while maintaining contact of all wheels with the surface thus allowing a good balance. The term "rocker" comes from the rocking aspect of the larger links on each side of the suspension mechanism. These rockers are connected to each other and the vehicle chassis through a differential gear mechanism. Relative to the chassis, when one rocker goes up, the other one goes down maintaining the chassis balance. One end of the Rocker is attached with the wheel assembly and the other end is attached to the Bogie. Bogie is similar to rocker but has driven wheel assemblies at both ends and forms the front end of the bot. A fork style arrangement for attachment of wheel assembly with the suspension system is used for a much rigid system and to minimize bending loads on the suspension pick-up points as shown in Figure 3 and Figure 4. This mechanism has many advantages like it maintains equal load on all wheels, has no axles or springs thus allows maintenance of equal traction on all wheels and can climb obstacles of height up to twice the diameter of wheels.
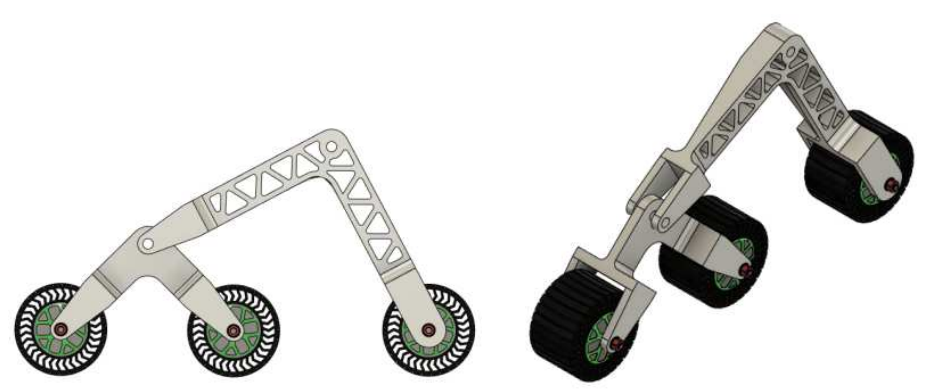

Figure 3: Rocker Bogie mechanism (Side View) Figure 4: Rocker bogie Mechanism (Isometric View)

\subsection{Wheel Design and Assembly}

\subsubsection{Tire}

There was a requirement for puncture proof tires that could easily be able to be an asset in movements in rough terrain. This tire model hence was inspired by michelin tubeless tire design. The angular rubber structure between the inner and the outer frame provides a cushioning effect as it can bend and come back to original form when varying load is applied acting like a spring system as seen in Figure 5. 


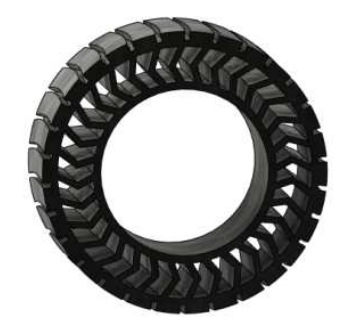

Figure 5: Wheel CAD Model

\subsubsection{In-wheel motor}

An in-wheel motor as shown in Figure 6 was selected for motion because of the compact assembly and its high efficiency. It has an outer rotor and a central stator normally used in E- Bikes. The motor has a power range of 300-700 W with peak of $1000 \mathrm{~W}$ and nominal $800 \mathrm{~W}$. The motor weight is $12 \mathrm{~kg}$ making it lightweight and an efficient selection. A speed of 100 - $400 \mathrm{RPM}$ is provided with torque of almost $80 \mathrm{Nm}$. This selection was done with main focus on these torque values which is essential in rough terrains. Since speed is a lesser important criterion with respect to force to pull which is provided by torque is essential. Final Assembly includes tire, in-wheel motor and a rim for aesthetic look. This whole assembly as shown in Figure 6 constitutes the wheel assembly. 6 individual wheel assemblies were installed to the rocker bogie arms which were further installed using a retaining nut. Being an individual motor mechanism also removes the requirement of a steering mechanism in the Bot.
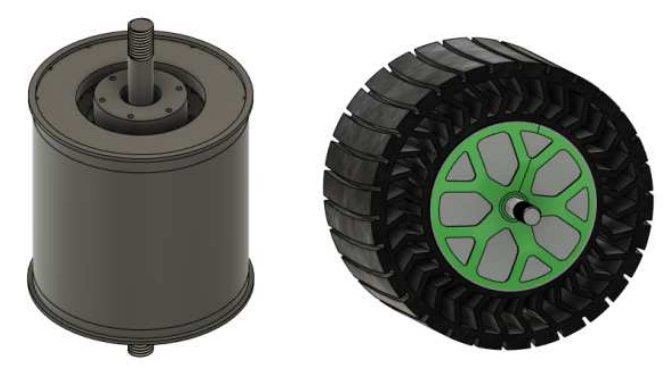

Figure 6: In-Wheel Motor (left) and Wheel assembly (right)

\subsection{Differential Gear Mechanism}

When seeing the Rocker Bogie mechanism, it always comes to mind that the body of the rover will either tip forwards or backwards until it hits the ground. The Differential here serves the purpose of keeping the Bot body at level. Relative to the body when one rocker side mechanism goes up the other goes down by the same level thus maintaining the body at halfway between the angles of two rockers. The working principle of Differential Gear involves a Three-Gear Mechanism meshed together where two Bevel gears as shown in Figure 7(a) are connected to the Rocker and the third central Bevel Gear shown in Figure 7(b) is attached inside the Bot body. All three gears are enclosed under a casing press-fitted with Roller bearings seen in Figure 8(a) to allow relative movement as shown in Figure 8(b). 


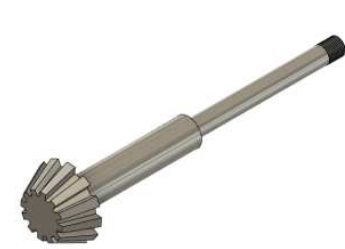

(a)

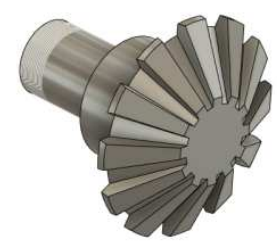

(b)

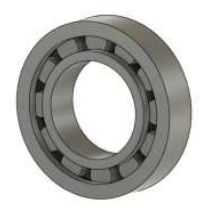

(a)

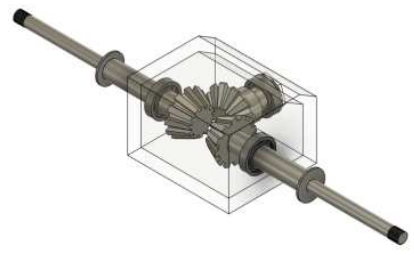

(b)

Figure 7: (a) Rocker Bevel Gear (b) Chassis Bevel Gear Figure 8: (a) Roller bearing ; (b) Differential Assembly

\subsection{Differential Gear Mounting}

For the bevel gear attachment to the rocker bogie mechanism an additional mounting has been used to prevent any bending of gear leading to failure as shown in Figure 9 and mounting is shown in Figure 10.
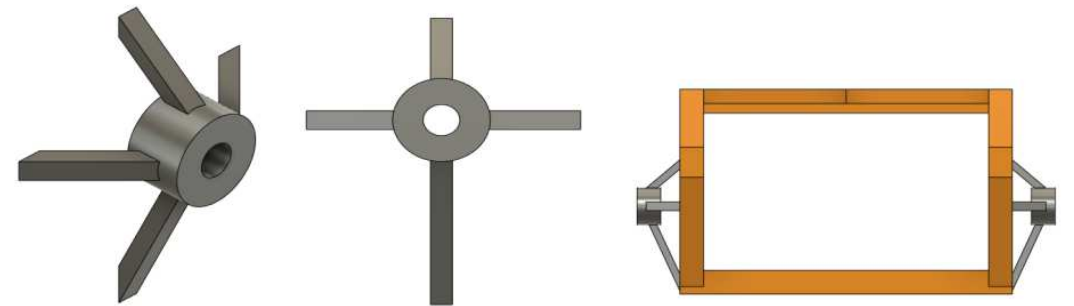

Figure 9: Isometric (left) and side view (right) of mounting Figure 10: Mounting position on chassis

\subsection{Robotic Arm}

The mechanical design of the robot arm is based on a robot manipulator with similar functions to a human arm [5]. Large companies have dedicated development teams and also the investment potential to implement such technologies [6]. This mechanism, as the name suggests, will perform all the functions that a human hand is expected to perform during a rescue operation be it moving huge debris away from the path as well as cutting live wires in order to reduce their contact length with the rescue workers. The working of the arm depends solely on electric motors which are fitted in every joint present in the arm. The arm is designed with a total of 3 arms with a rotating Jaw at the end. The arm is designed in a way so that when in rest mode, it is in perfect compact mode with a collapsed length of $201 \mathrm{~mm}$ as shown in Figure 12 and maximum extended length of about 1000mm as shown in Figure 11.
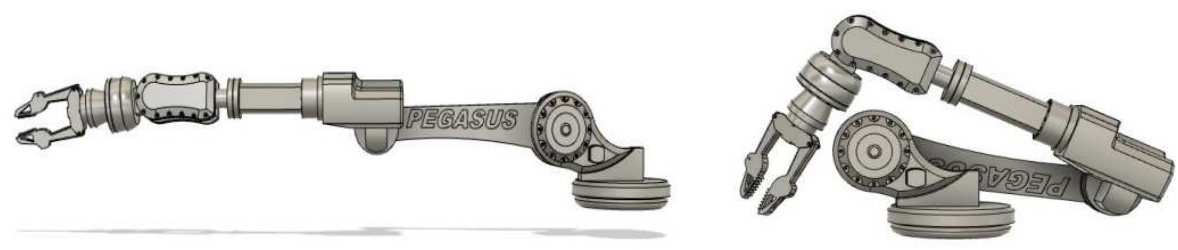

Figure 11: Fully Extended Robotic arm Figure 12: Collapsed View (Robotic arm)

\subsection{Loader Bucket Mechanism}

For low lying debris and obstacles present near the lower chassis Bot cannot solely rely on the robotic arm to reach out such places and remove the load. So, a loader bucket mechanism is incorporated, which is a combination of a slider, 
revolution and lateral movement to ensure that these obstacles are removed from the path of the bot. The Loader Bucket mechanism comprises of 3 parts i.e. Bucket as shown in Figure 13 which helps in the removal of obstacles and in lifting up the debris in front. The Loader arm as shown in Figure 14 being the second part which does the operation of carrying the load and moving to and fro as this is the arm which holds the bucket as well as the motor rotating the bucket, and also is the key in the oscillatory front and back movement. Slot mechanism as shown in Figure 15 is the third part which is a rigid combination of two structures which houses one heavy duty metal gear servo motor each to enable the lateral movement of the loader arm. Fully extended length of the Loader Arm mechanism is 925mm as shown in Figure 16.

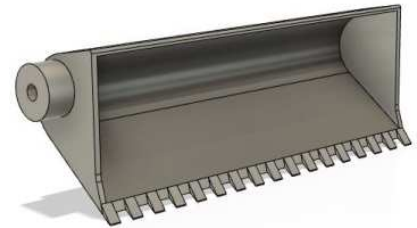

Figure 13: Bucket

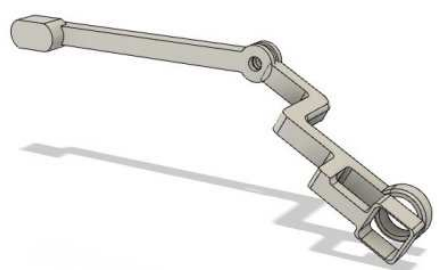

Figure 14: Loader Arm

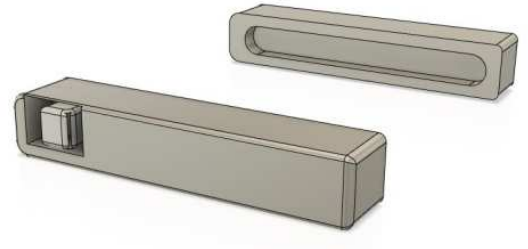

Figure 15. Slot Mechanism

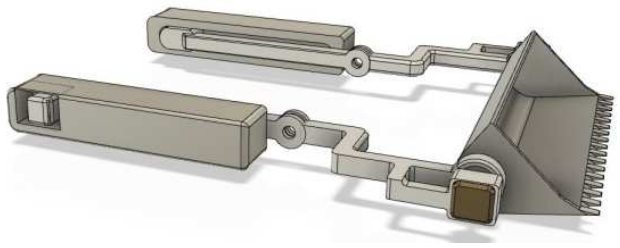

Figure 16: Loader Bucket Mechanism Assembly

\subsection{Panels for Robotic Arm}

The servo powered panels can open and close autonomously hence protecting inner components while allowing the robo arm to get in full stretch when required as shown in Figure 17.

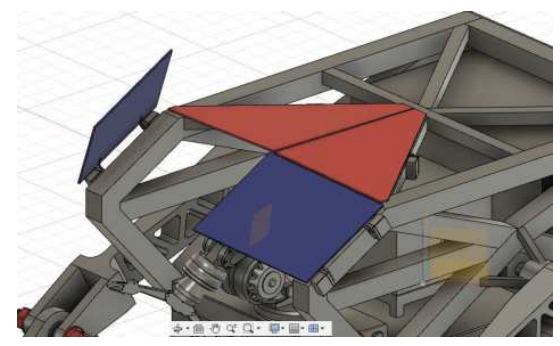

Figure 17: Isometric view of panel attachment on chassis member

\section{MANUFACTURING}

\subsection{Tubular Space Frame}

The manufacturing process begins with separating each tube designed separately as a file and measuring their lengths. Then each tube's length is measured and are marked on the 5 meter tubes. After marking each tube is cut according to their grooves using a Miter Saw. A Jig is constructed which is used as a mold which helps in accurate positioning and placement 
of chassis tubes so that they can be welded together. TiG welding is used for joining the tubes together as this welding process is much more accurate and concentrated as compared to normal Arc welding. For the prevention of corrosion on the structure Powder coating was done on the tubes to protect them from the corroding environment. Different views are shown in Figure 18 and Figure 19.
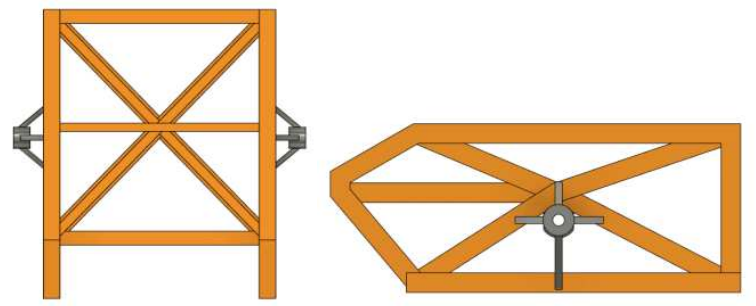

Figure 18: Top View Figure 19: Side View

\subsection{Rocker-Bogie Mechanism}

As the Rocker and Bogie linkages have dimensions which are not feasible for being machined from a block of metal because it will lead to a lot of wastage of raw material, therefore the casting process is adopted in which the basic outer structure of the linkages are casted using green sand casting process. Then, these casted parts are machined via 5-axis CNC machine to provide further details and better surface finish. The Wheel assembly bolting points, rocker bogie common points and rocker point for mounting to the chassis are thus machined later after the casting process is over.

\subsection{Differential Gear Mechanism}

A circular billet of required dimensions is taken and 3-D CNC machining is used for reduction in diameter for respective areas and proper positioning of teeth grooves so that there was minimal presence of Backlash when mating with other bevel gears. The thread forming of the gears is done on a Lathe machine according to the specifications of the retaining nuts and locker nut. As the bevel gears will be subjected to large forces, therefore surface treatment is performed on the bevel gear using the process of Carburizing. For the differential casing manufacturing standard thickness sheets Aluminium 6061-T6 are used which are cut to the required dimensions using Waterjet machining and machining for Roller bearing slots is done using the same process. After the proper press fit of bearing and placement of bevel gears the different parts of the casing are welded together to make an enclosed box.

\subsection{Differential Gear Mounting}

These are 3-D CNC machined from solid blocks of metal and for the attachment of these to the chassis the solid standard machined billets are used which provide structural support. They are welded to the Chassis body using TiG welding.

\subsection{Wheel Assembly}

The given wheels are proposed to be manufactured via additive manufacturing techniques. The method adopted for demonstration is to use TPU 95A for simulation on Ultimaker which shows 7 days but the actual prototype developed by michelin uses natural rubber with glass fibre reinforcement. In 2017 at Movin'On, Michelin unveiled its VISION concept tire to advance mobility through four main innovation pillars: Airless, Connected, 3-D printed or rechargeable tread pattern, and $100 \%$ sustainable (renewable or bio-sourced materials). We have tried to use the same concept into this prototype design. Further the motor is a bought part and assembly requires installation of tires on hub motors and attaching 
rims manufactured using punching and blanking technique.

\subsection{Robotic Arm}

Jaw 1 and the base as shown in Figure 20 are casted and joined together using braze welding. The square cut for motor placement is drilled using the Mortiser's feed lever. Jaw 2 as shown in Figure 21 is casted in two parts using Cast Iron as the base material and nickel and chromium as alloy materials. A core is used to provide a hollow structure in order to incorporate the wirings of the motor and controller. After casting, the core is removed and the two parts are joined using braze welding. Jaw 3 as shown in Figure 22 is also manufactured in the similar way of Jaw 2. AISI 316 has been chosen for casting of different components of Rotating Jaw as shown in Figure 23 due to its non-magnetic character and the austenitic phase after casting. The whole part is separated into 3 major parts for manufacturing the upper jaw is manufactured using a two-part mold using core. The middle jaw is an oscillating part directly machined from a solid stainless-steel block. The third part, fork jaws are machined from a solid steel block to maintain the structural integrity as well as toughness. After all the parts are manufactured and joined properly, there is a chance of the robotic arm getting exposed to various fluids during the rescue operation. Stainless Steel 300 Coating is used because it is highly resistant to corrosion as it has AISI 316L Alloy Flake pigments present in it and also acts as a great moisture barrier.

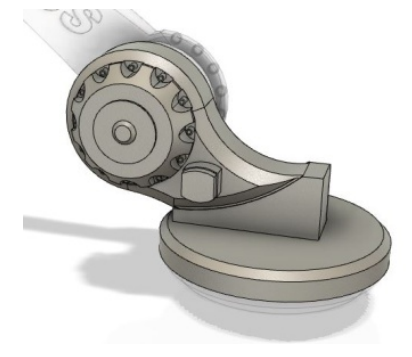

Figure 20: Jaw 1

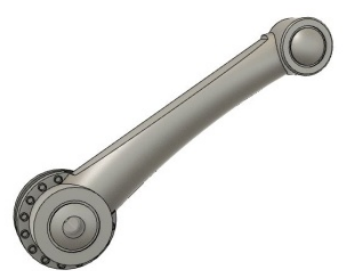

Figure 21: Jaw 2

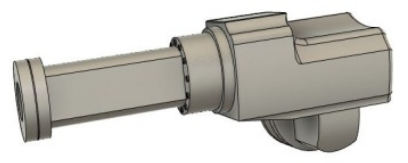

Figure 22: Jaw 3

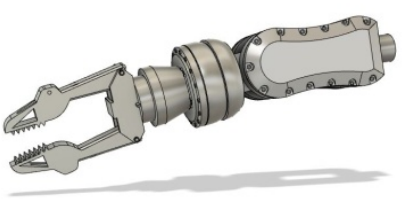

Figure 23: Rotating Jaw

\subsection{Loader Bucket Mechanism}

Bucket is manufactured using a carbon steel sheet and die forging is using a curved shaped die to make the depression present in the bucket, where die forging ensures less manufacturing cost. Once the shape is obtained, the obtained sheet is then heat treated to obtain a hard microstructure overall. The side walls are machined from a single sheet and welded on both the sides using MIG welding. For the teeth, amalgam of methods are performed including rough forging using a $\mathrm{T}$ type through an air hammer, performing coarse crushing, heating the ingot, and stamping to get the desired shape. The Loader Arm is manufactured in two parts, the major structure attached to the bucket on one side and to the slot mechanism on the other side, and the other part which will hold the servo motor for bucket rotation. Both the parts are made of carbon steel, and the basic structure is casted. After the casting is complete, these two parts are welded together using Braze welding for a seamless join and then sanding is performed. The slot mechanism for to and fro motion is purchased and the 
housing here is casted in two parts and then welded together. Hardide coating is applied to the whole assembly as it is placed under the bot chassis where there is a maximum chance of getting in contact with some debris material which might result in deterioration of the material.

\subsection{Panels for Robotic Arm}

The desired cut out of panels from a $3 \mathrm{~mm}$ thick sheet of transparent polycarbonate using a Miter saw and then the edges are sanf to make it smooth.

\subsection{Additive Manufacturing}

The parts which were additively manufactured were Roboarm rotating base, Camera stands as shown in Figure 24. The printer specifications used were as follows:

Printer Speed - 50 mm/sec, Infill Density - 30\%, Infill Pattern - Concentric, Layer Height - 0.15mm, Material Silver Metallic PLA, Nozzle size $-0.4 \mathrm{~mm}$, Printing Temperature $-205^{\circ} \mathrm{C}$, Build Plate Temperature $-60^{\circ} \mathrm{C}$

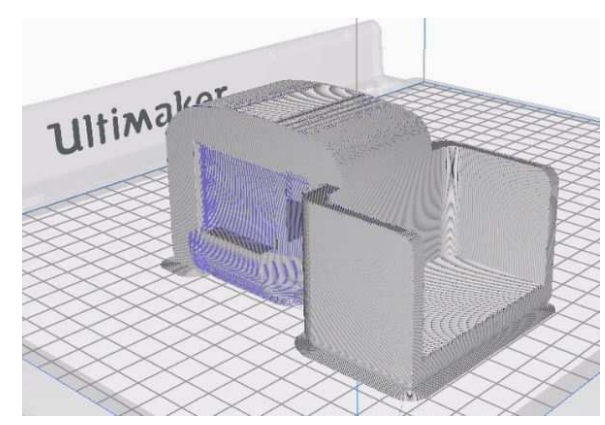

Figure 24: Additive manufacturing simulation using UltimakerCura

\section{CAlculations}

\subsection{Rocker Bogie Mechanism Calculations}

There will be uneven rocky terrain due to bridge collapse for which we assume following conditions i.e. can climb obstacles upto $450 \mathrm{~mm}$ height (like stones, concrete, etc.) and length of upto 1000m, Can climb and move at an angle of upto 45 degrees

\subsubsection{Calculations of Bogie Linkages}

Tire diameter $=250 \mathrm{~mm}$

The bogie Linkage length should be such that there is no linkage striking the obstacle while climbing, therefore taking the distance between centers as $500 \mathrm{~mm}$ as seen in Figure 25. This length is then used to calculate the linkage length as follows: It follows Pythagoras theorem, 


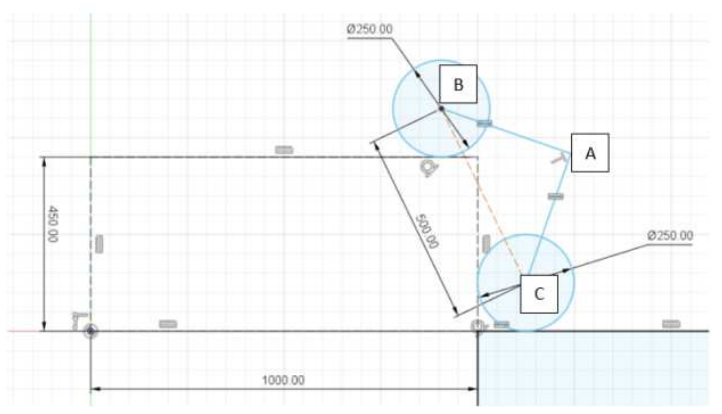

Figure 25: Bogie Linkage Calculation Drawing I

$\mathrm{B}$ and $\mathrm{C}$ represent the centers of tires, $\mathrm{ABC}$ is a right-angled triangle for which:

$$
500^{2}=A B^{2}+A C^{2}
$$

$$
\mathrm{AB}=\mathrm{AC}=\mathrm{x}
$$

Therefore, $\mathrm{x}=350 \mathrm{~mm}$

\subsubsection{Calculations of Rocker linkages}

First two-wheel pairs should be placed at horizontal position. Third wheel pair should nearly complete its rising before starting the rising of the first pair of wheels. By placing the wheel in such a manner, we obtained dimension of linkages as seen in Figure 26.

The distance between tire centers was found to be approx. $840 \mathrm{~mm}$.

Triangle ADE is a right-angled triangle, therefore by Pythagoras theorem:

$$
840^{2}=A D^{2}+D E^{2}
$$

$\mathrm{AD}=\mathrm{DE}=\mathrm{y}$

Therefore, $\mathrm{y}=595 \mathrm{~mm}[7]$

Final configuration of Rocker Bogie mechanism is calculated and shown in Figure 27.
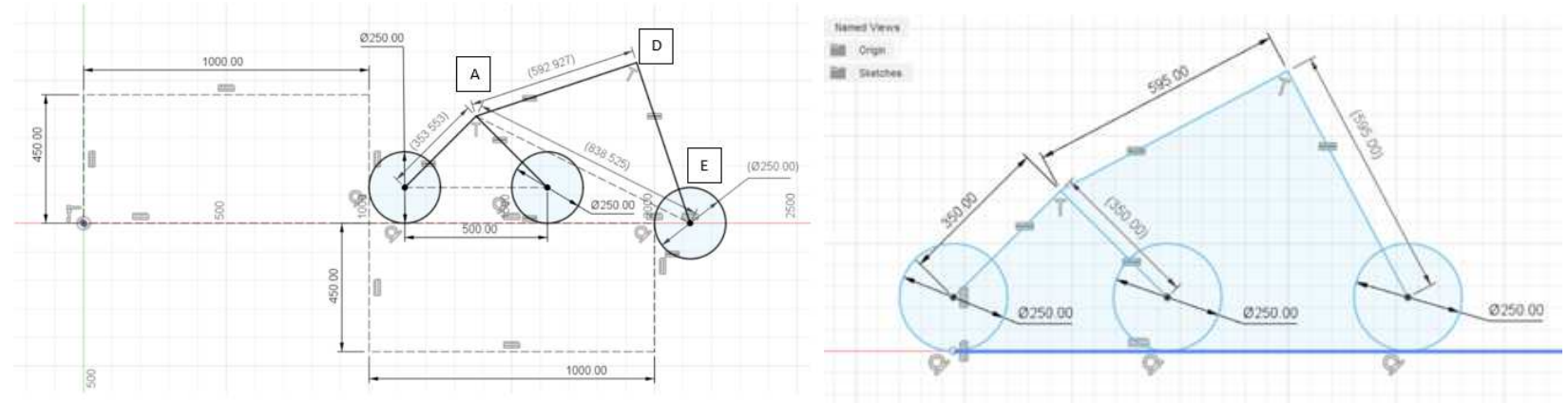

Figure 26: Bogie Linkage Calculation Drawing II Figure 27: Final Rocker-Bogie Configuration Drawing

\section{SENSORS AND INPUTS}

\subsection{Battery}

The battery used is a GTK Lifepo 4 which is a $12 \mathrm{~V} 800$ AH lithium battery with BMS $12.8 \mathrm{~V}$ for caravan RV EV inverter It 
also has a solar charger hence when on site 2 batteries can be taken once one battery drains we can shift to the other battery while the drained battery can recharge using solar charger at the docking station hence the bot can be used for a longer period of time.

\subsection{Stereo-Vision Camera}

A stereo camera is a type of camera with multiple lenses each having a unique image sensor or film frame. It looks similar to a biological eye because it uses the same image perception technique and creates a similar binocular vision. The Omega Stereo Camera from ARCURE is being used as it provides excellent operating conditions ranging from -45 degree to 75 degree celsius. It consists of an inbuilt CMOS sensor which helps in the generation of the digital image. The Stereo-Vision camera is a bought part but the Revolving stand has been 3-D Printed in accordance with camera specifications.

\subsection{LiDAR}

LiDAR stands for Light Detection and Ranging, is a method for measuring distances (ranging) by illuminating the target with laser light and measuring the reflection with a sensor. Differences in laser return times and wavelengths can then be used to make digital 3-D representations of the target. It has terrestrial, airborne, and mobile applications. LiDAR is used here for generating 3D maps of the surrounding area for object detection necessary for perception signals for Object Detection and Response. The LiDAR named Velodyne Ultra Puck is used which provides 200m range, Horizontal $360^{\circ}$ and Vertical $40^{\circ} \mathrm{FoV}$ (Field of Vision).

\subsection{Inertial Measurement Unit (IMU)}

Inertial measurement unit is a device that measures and informs about a body's precise force, angular rate, and sometimes the alignment of the body using an amalgamation of accelerometers, gyroscopes and magnetometers. The accelerometer measures the velocity and acceleration, Gyroscope measures rotation and rotational rate and Magnetometer establishes directional heading. The main purpose of the IMU is to help the onboard computer to calculate the altitude and relative position to a reference frame of a bot. In addition to this, IMU will serve as a supplement to the GPS for estimating Bot position.

\subsection{Range-R}

It is a Through wall Human detection system. One of the primary reasons for such rescue operations is to obtain signs of life, if any and then work towards extricating them. It is way too risky and a time consuming task to clear the whole area while scanning for signs of life and to overcome this Range-R system has been incorporated which has been in mass production for military purposes as well as for fire Fighters during rescue operations. RADAR here, due to its high sensitivity, can detect human breathing through a thick, solid concrete wall. The sensors operate in the frequency range of 1-10 GHz because this range is effective in penetrating through solid structures such as concrete, wood and plastic. Motion detection is based on the doppler shift principle, where the wave return time is measured to evaluate the distance between the object and the equipment and at continued intervals. A combination of SONAR and RADAR is used to identify objects/living creatures under the debris. RANGE-R uses a high frequency radio wave working on the principle of SONAR, measures the distance of the solid object whereas RADAR can penetrate through the walls and detect human life signatures but for a short distance.

\section{SOFTWARE ARCHITECTURE}




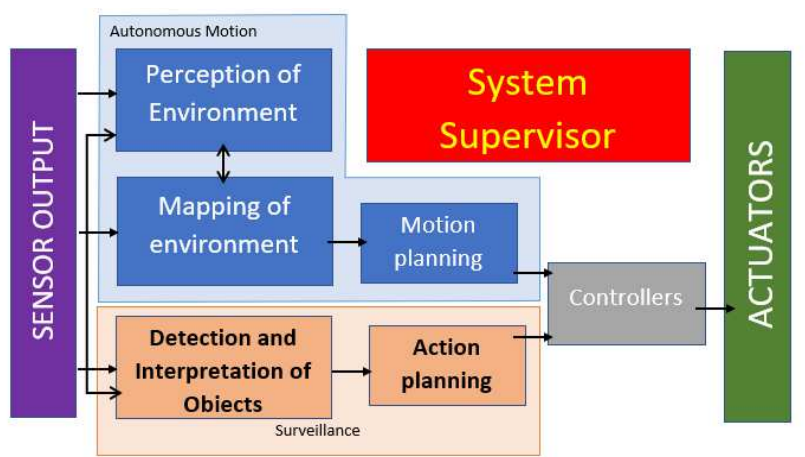

Figure 28: Plan Map (Software Integration)

While this overview of the software stack as shown in Figure 28 will not provide implementation detail, it should give you a good understanding of all of the software components required to make the bot function which has been derived from [8]. The given higher rank Software architecture describes different sub parts of the overall software hierarchy. These are broadly divided into 5 sections which are Sensor Output, Autonomous Motion, Surveillance, Actuation, System Supervisor.

\subsection{Sensor Output}

These are the sensor data which is gained from various sensors incorporated in the bot which is also converted further to data of the form interpretable by other modules of the software.

\subsection{Autonomous Motion}

This part specifically deals with the bots movement and behaviour autonomously. It is further divided into Environment perception, Environment Mapping and motion planning.

\subsubsection{Perception of Environment}

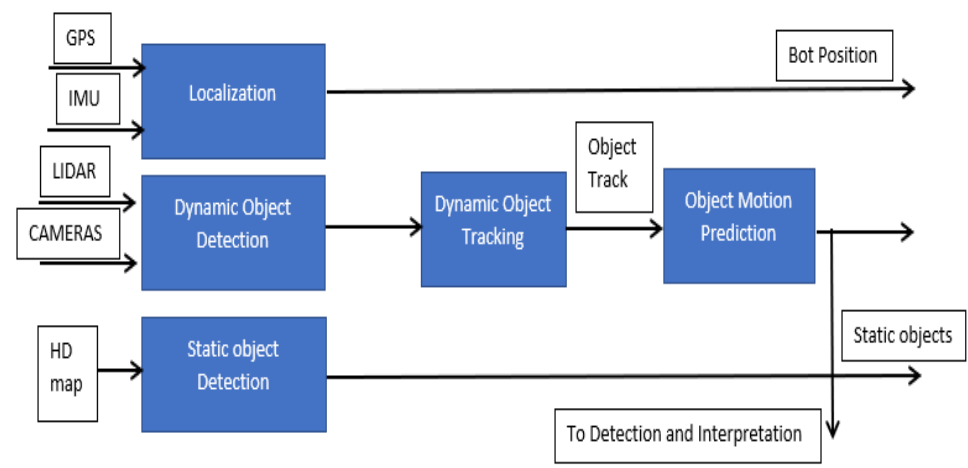

Figure 29: Perception environment software structure plan map

There are two important parts of the perception stack, localizing the bot in space, as well as classifying and locating the important elements of the environment as shown in Figure 29. The localization module used multiple sources of information, such as the GPS location, IMU calculations. It then combines all the outputs to provide an accurate vehicle location. For much better precision, some localization modules also integrate data from LiDAR and camera. Generally, the classification and localization problem of the environmental elements is divided into two segments i.e. detecting dynamic and static objects in the environment. The dynamic object detection module uses inputs from a set of cameras as well as 
LiDAR point clouds to create 3D bounding boxes around dynamic objects in the environment. The 3D bounding boxes detect the class and type of object along with the exact position, orientation and size of the object.

\subsubsection{Mapping of Environment}

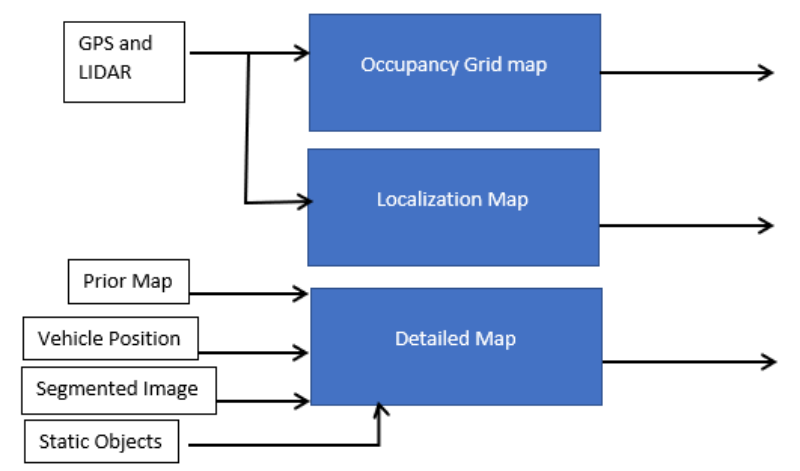

Figure 30: Mapping environment software structure map

The occupancy grid is a map of all static objects in the environment surrounding the bot. LIDAR is predominantly used to construct the occupancy grid map. A set of filters are first applied to the LIDAR data to make it usable by the occupancy grid. The localization map, which is constructed from LIDAR, or camera data, is used by the localization module in order to improve bot state estimation. Sensor data is compared to this map while moving to determine the motion of the bot relative to the localization map. This motion is then combined with other proprioceptor sensor information to accurately localize the bot. The detailed map is a combination of pre-recorded data as well as incoming information from the current static environment gathered by the perception stack as shown in Figure 30.

\subsubsection{Motion planning}

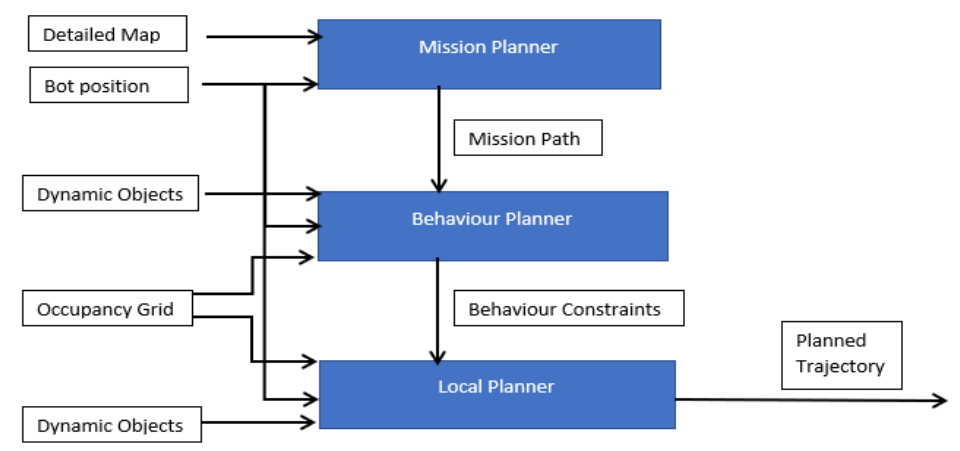

Figure 31: Software plan map for motion

The motion planner handles long term planning and defines the motion over the entire horizon of the task, from the current location, through the network to its final destination. To find the complete mission, the motion planner determines the optimal sequence of segments that connect origin and destination, and then passes this to the next layer. The Behavior planner is the next level of abstraction, to address short term planning problems. The behaviour planner is responsible for establishing a set of safe actions or manoeuvres to be executed while travelling along the mission path as shown in Figure 31.

\subsection{Surveillance}




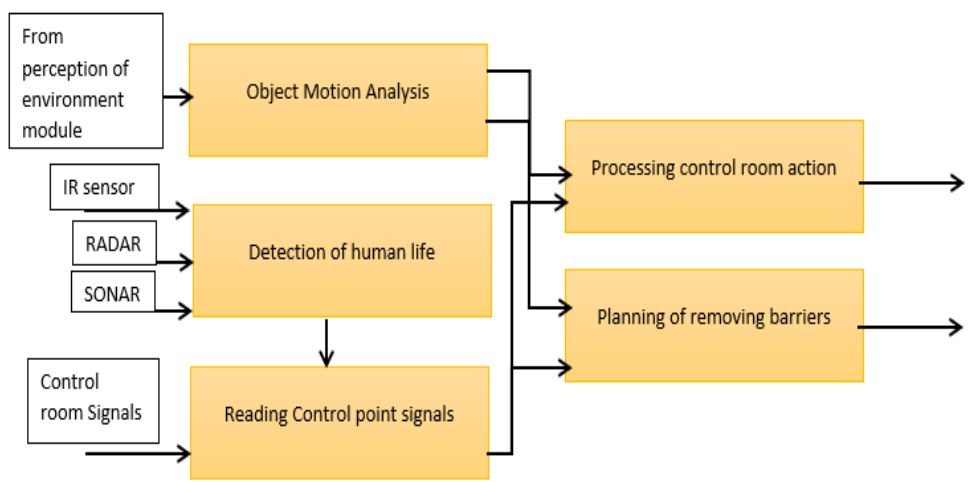

Figure 32: Surveillance software structure

The data taken from perception of the environment module provides data for object motion analysis to determine how to tackle the dynamic object. Also, motion analysis is used to differentiate among the types of dynamic objects for detection of life. Further IR and RADAR data are taken for determining human life which is further transferred to reading control point signals module. The reading control point signal module is in direct contact with the control room providing on site data and object detection data for further signals on instructions to wait for the team to come or to start removing boulders and barriers in between. Further moving on to action planning which decides on the processed signal which action to take place. It interprets data and further sends signals to controllers for specific actuation as shown in Figure 32.

\subsection{Actuation}

Controllers govern the amount of actuation of actuators here they consist of servo motors, in wheel motors and other electronic and mechanical output devices.

\subsection{System Supervisor}

The system supervisor is the module that continuously monitors all aspects of the autonomous bot and gives the appropriate warning in the event of a subsystem failure. There are two parts, the hardware supervisor, and the software supervisor. The hardware supervisor continuously monitors all hardware components to check for any faults, such as a broken sensor, a missing measurement, or degraded information. while the software supervisor validates the software stack and ensures all elements' smooth functioning.

\section{CONCLUSIONS}

This overall design concept hence includes all the necessary provisions for the bot to be further addressed for implementation as an autonomous gadget having the following key highlights :-

- Fully functional Robotic Arm

- Heavy duty Loader bucket system

- $\quad$ Rocker-Bogie mechanism for easy motion in rough terrain

- $\quad$ Puncture proof tire design

- $\quad$ Autonomous Motion based design (including all required sensors).

All these have been also designed in accordance to manufacturing which has been defined and includes traditional 
to new age manufacturing processes like Casting, $\mathrm{CNC}$ and additive manufacturing.

This work can be further extended to surveillance domain by incorporating changes of making the design changes of removing loader arm and robotic arm with incorporation of camouflage look as per assigned place of use for which an iteration has been shown in Figure 33. This extends the implementation of concept from disaster mitigation to border surveillance and defence. Also work can be done to make a testing prototype for the same by working on making the software architecture into a practical software design for the given concept.

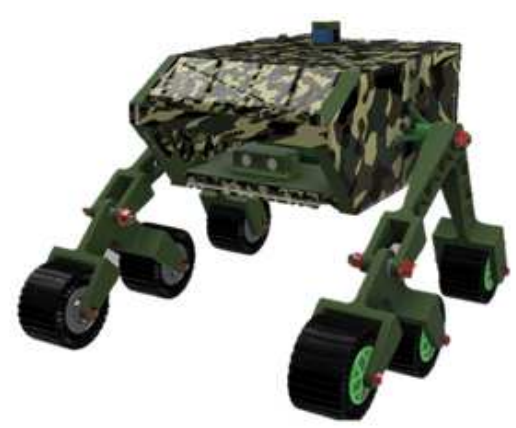

Figure 33: Bot - Surveillance version

\section{REFERENCES}

1. Maza, I., Viguria, A., \&Ollero, A. (2006). Networked aerial-ground robot system with distributed task allocation for disaster management. In Proceedings of the IEEE International Workshop on Safety, Security and Rescue Robotics.

2. Kuntze, H. B., Frey, C. W., Tchouchenkov, I., Staehle, B., Rome, E., Pfeiffer, K.,...\&Wöllenstein, J. (2012, November). SENEKA-sensor network with mobile robots for disaster management. In 2012 IEEE Conference on Technologies for Homeland Security (HST) (pp. 406-410). IEEE.

3. SATTAR, MD ABDUS. "CORPORATE SOCIAL RESPONSIBILITY IN DISASTER RISK MANAGEMENT: A STUDY ON SOME SELECTED PRIVATE COMMERCIAL BANKS OF BANGLADESH." International Journal of Environment, Ecology, Family and Urban Studies 7.5 (2017): 7-18.

4. Schmid, K., Tomic, T., Ruess, F., Hirschmüller, H., \&Suppa, M. (2013, November). Stereo vision based indoor/outdoor navigation for flying robots. In 2013 IEEE/RSJ International Conference on Intelligent Robots and Systems (pp. 3955-3962). IEEE.

5. Casper, J. L., \& Murphy, R. R. (2002, May). Workflow study on human-robot interaction in USAR. In Proceedings 2002 IEEE International Conference on Robotics and Automation (Cat. No. 02CH37292) (Vol. 2, pp. 1997-2003). IEEE.

6. MALISUWAN, SETTAPONG, and WASSANA KAEWPHANUEKRUNGSI. "Ad Hoc UAV LTE Networks for Critical Communications." International Journal of Computer Networking 6.1 (2016).

7. Qi, A. N. W., Voon, K. L., Ismail, M. A., Mustaffa, N., \& Ismail, M. H. Design and Development of a Mechanism of Robotic Arm for Lifting Part1.

8. Francalanza, E., Fenech, A., \&Cutajar, P. (2018). Generative design in the development of a robotic manipulator. Procedia CIRP, 67, 244-249.

9. Chinchkar, D., Gajghate, S., Panchal, R., Shetenawar, R., \&Mulik, P. (2017). Design of Rocker Bogie Mechanism. International Advanced Research Journal in Science, Engineering and Technology, 4(1), 46-50. 
10. Tuzzohora, F. A. T. E. M. A., S. A. J. I. B. Parvez, and S. H. A. H. R. I. A. R. Rahman. "Effective evacuation management and mitigation plan for earthquake: A case study on Lalbagh area of Dhaka city." International journal of earthquake engineering and geological science 5.2 (2015): 1-16.

11. Bussemaker, K. J. (2014). Sensing requirements for an automated vehicle for highway and rural environments.

12. Thakur, P.,(March 11, 2015) Disasters cost India \$10bn per year: UN report. Retrieved from https://timesofindia.indiatimes.com/india/Disasters-cost-India-10bn-per-year-UN-report/articleshow/46522526.cms

13. Kushwah, Virendra Singh, Sandip Kumar Goyal, and PriushaNarwariya. "A survey on various fault tolerant approaches for cloud environment during load balancing." Int J ComputNetwWirel Mobile Commun 4.6 (2014): 25-34.

\section{APPENDIX - 1.1. COST JUSTIFICATION/ANALYSIS}

UN report says $\$ 9.8$ billion per year expenditure on disaster management in India [9]. Our Bot costs about 9 lakhs INR which is about $\$ 11,900$ for each robot This is about $0.00012 \%$ of the total loss India faces yearly on disasters. With one bot easily having a life cycle of more than 10 years for all parts and 5 years for battery life we can say that this reusability provides a value for money and reduces cost in the long term. Also costing is done for the prototype taking single part manufacturing cost for individual parts which can be further reduced by an easy margin of $30-40 \%$ if mass production of the product takes place.

\section{APPENDIX - 1.2. FINAL DESIGN}

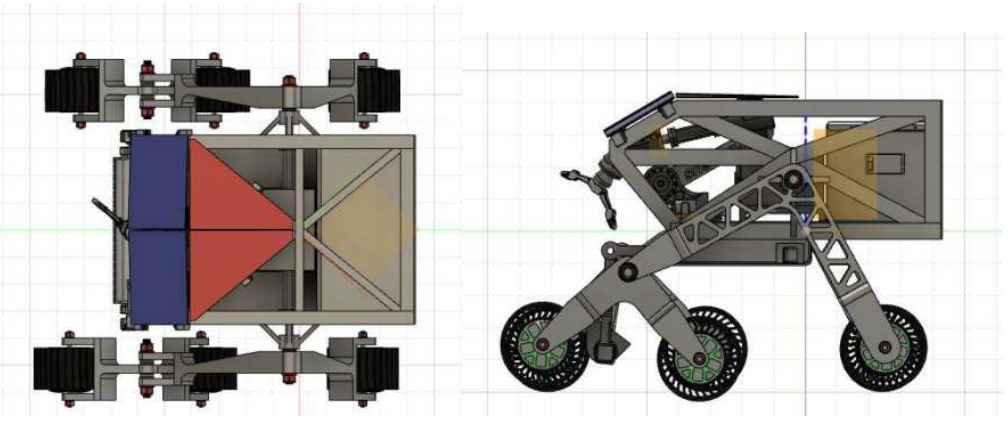

Figure A.3. Top View (Without Body Panels) Figure A.4. Side View (Without Body Panels)
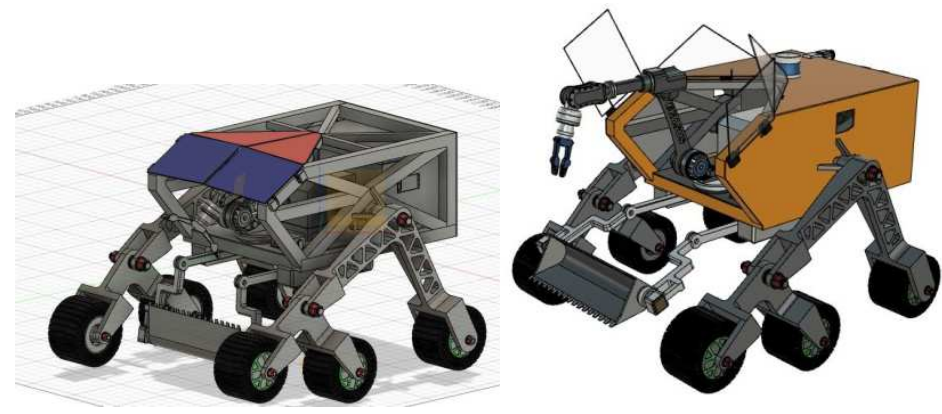

Figure A.5. Isometric View (Without Body Panels) Figure A.6. Isometric view with body panels 